\title{
LATTICE DYNAMICS OF ALKALI METALS IN A THREE-BODY INTERACTION
}

\author{
A.A. Coelho \\ Instituto de Fisica, UNICAMP, C.P. 6165, 13081 Campinas, SP, Brasil \\ AND M.M. SHUKLA \\ Departamento de Fisica, UNESP, C.P. 473, 17033 Bauru, SP, Brasil
}

(Received March 23, 1994)

\begin{abstract}
The original model of Das et al. is modified in extending the electron-ion interaction on a three-body forces and including the crystal equilibrium condition to reduce one independent parameter. We studied the phonon dispersion relations along the three principal symmetry directions i.e. $[\xi, 0,0]$, $[\xi, \xi, 0]$ and $[\xi, \xi, \xi]$ and $\theta-T$ curves of alkali metals, $\mathrm{Na}, \mathrm{K}, \mathrm{Rb}, \mathrm{Cs}$ and $\mathrm{Li}$. There is close agreement between the computed results and the experimental observations.
\end{abstract}

PACS numbers: 63.20.-e, 65.40.-f

\section{Introduction}

Theoretical study of lattice dynamics of cubic metals has been a subject of comprehensive study for the past couple of decades. The availability of the experimental phonon dispersion relations along the principal symmetry directions had played a vital role to test the validity of the theoretical models. There are two broad categories of models, i.e. one on first principles initiated by Toya [1] and the other on phenomenological basis initiated by de Launay [2]. The models on first principles are very difficult to handle physically and mathematically. The models on phenomenological basis are very simple to handle with and to a certain extent reproduce the experimental results on phonons closer or better than given by the models on first principles. In the present work we would study the recent phenomenological model of Das et al. [3]. The model of Das et al. [3] consists in considering the interionic interaction by a two-body pair potential and the electronic interaction on an effective three-body interaction. The general features and theoretical details of this model for cubic metals and for hep metals have been given by Sarkar et al. [4] and Dutta et al. [5]. We would confine here our 
study for bcc metals. While Sarkar et al. [6] studied the electronic interactions in bcc metals up to second nearest neighbours (there is an error introduced by them in their work), we have extended such an interaction out to third neighbours to see if the electronic part of the dynamical matrix has some definite influence on the calculation of the phonon frequencies in bcc metals. We introduced also the crystal equilibrium condition of Upadhyaya et al. [7] to reduce one independent model parameter. The study is confined to five alkali metals by computing the phonon dispersion relations along the principal symmetry directions and the $\theta-T$ curves of them. A comparison with the experimental results forms the subject matter of this paper.

\section{Theory}

The details of the theory is given by Das et al. [3]. Here we will give only the necessary modifications. The total potential energy of the crystal is given by

$$
\Phi=\frac{1}{2} \sum_{l, l^{\prime}} \Phi\left(l^{\prime}, l\right)+E(\vartheta)
$$

In Eq. (1) $\Phi\left(l, l^{\prime}\right)$ represents the two-body pair potential for the ion-ion interaction and $E(\vartheta)$ is the volume dependent potential which describes the electron-ion interaction. By expanding $E(\vartheta)$ in terms of the equilibrium volume $\vartheta_{0}$, we have

$$
E(\vartheta)=E(\vartheta)-p_{\mathrm{e}}\left(\vartheta_{0}\right) \chi+\frac{1}{2} K_{\mathrm{e}} \vartheta_{0} \chi^{2} .
$$

Lax [7] has pointed out that the only important term in Eq. (3) is the one which is multiplied by $K_{\mathrm{e}}$, the other two do not contribute to the dynamical matrix.

Following the schemes of Das et al. [3] the average volume strain in the $l$-th cell of a bcc lattice extending out to third neighbours is given by

$$
\begin{aligned}
\left\langle\chi^{l}\right\rangle & =\frac{3}{n_{1}} \sum_{l_{1}, \alpha} \frac{r_{\alpha}\left(l, l_{1}\right) u_{\alpha}\left(l_{1}\right)}{r^{2}\left(l, l_{1}\right)}+\frac{3}{n_{2}} \sum_{l_{2}, \alpha} \frac{r_{\alpha}\left(l, l_{2}\right) u_{\alpha}\left(l_{2}\right)}{r^{2}\left(l, l_{2}\right)} \\
& +\frac{3}{n_{3}} \sum_{l_{3}, \alpha} \frac{r_{\alpha}\left(l, l_{3}\right) u\left(l_{3}\right)}{r^{2}\left(l, l_{3}\right)} .
\end{aligned}
$$

In the work on lithium by Sarkar et al. [6] there was an error introduced in the calculation of $\left\langle\chi^{l}\right\rangle$. The right hand side of Eq. (2) of that paper was divided by half which is wrong. This mistake was further performed by them in their Eqs. (4) and (5) also. This fact can be easily verified by their other work (Sarkar et al. [4]) where they considered the dynamical matrix for the first neighbour interaction only. In Eq. (3), we have that $n_{3}$ is the coordination number of third neighbour of $l$ and $r\left(l, l_{3}\right)$ is the distance between the atom at $l$ and atom $l_{3}$. The electron-ion dynamical matrix is given by

$$
D_{\alpha \beta}^{\mathrm{e}}=E_{2}[\exp (\mathrm{i} q \cdot r-\omega t)],
$$

where $E_{2}$ in terms of the electronic strains is given by

$$
E_{2}=\frac{1}{2} K_{\mathrm{e}} \vartheta_{0} \sum_{l}\left(\chi^{l}\right)^{2}
$$


We deviated from Sarkar et al. [6] in evaluating the sum $\sum \chi^{l}$. We first made $\chi^{l}=\chi_{1}^{l}+\chi_{2}^{l}+\chi_{3}^{l}$ summing the average strain for the first, second and third nearest neighbours and then squared it, i.e.

$$
E_{2}=\frac{1}{2} K_{\mathrm{e}} \vartheta_{0} \sum_{l}\left[\chi_{1}^{l}+\chi_{2}^{l}+\chi_{3}^{l}\right]^{2},
$$

our results of $E_{2}$ dropping out the term for the third neighbour, i.e. $\left\langle\chi^{7}\right\rangle_{3}=0$ tallied with the expression of $D_{\alpha \alpha}^{3}$ and $D_{\alpha \beta}^{3}$ of Eq. (5) of Sarkar et al. [6] multiplied by 4 as they had divided Eq. (2) of their paper by a factor of half. The typical diagonal and non-diagonal part of the dynamical matrix is given by

$$
\begin{aligned}
D_{\alpha \alpha}^{3}= & 2 a K_{\mathrm{e}}\left[S_{\alpha}\left(C_{\alpha}+C_{\beta} C_{\gamma}\right)+\frac{1}{8} S_{2 \alpha}\left(C_{2 \beta}+C_{2 \gamma}\right)\right]^{2}, \\
D_{\substack{\alpha \beta \\
\alpha \neq \beta}}^{3}= & 2 a K_{\mathrm{e}}\left[S_{\alpha}\left(C_{\alpha}+C_{\beta} C_{\gamma}\right)+\frac{1}{8} S_{2 \alpha}\left(C_{2 \beta}+C_{2 \gamma}\right)\right] \\
& \times\left[S_{\beta}\left(C_{\beta}+C_{\alpha} C_{\gamma}\right)+\frac{1}{8} S_{2 \beta}\left(C_{2 \alpha}+C_{2 \gamma}\right)\right] .
\end{aligned}
$$

Following Sarkar et al. [6], we considered the interionic interaction in bcc metals extending out to first two neighbour interaction. Thus the expression of $D_{\alpha \alpha}^{2}$ and $D_{\alpha \beta}^{2}$ are the same as given by Eq. (7) in the paper of Sarkar et al. [6].

The expression for the typical diagonal and non-diagonal part of the total dynamical matrix, ion-ion part as well as the electron-ion part, is given by

$$
\begin{aligned}
D_{\alpha \alpha} & =\frac{8}{3}\left(\phi_{1}^{\prime \prime}+2 \phi_{2}^{\prime}\right)\left(1-C_{\alpha} C_{\beta} C_{\gamma}\right)+4 \phi_{2}^{\prime \prime} S_{\alpha}^{2}+4 \phi_{2}^{\prime}\left(S_{\beta}^{2}+S_{\gamma}^{2}\right)+2 a K_{\mathrm{e}} \\
& \times\left[S_{\alpha}\left(C_{\alpha}+C_{\beta} C_{\gamma}\right)+\frac{1}{8} S_{2 \alpha}\left(C_{2 \alpha}+C_{2 \gamma}\right)\right]^{2}, \\
D_{\alpha \beta} & =\frac{8}{3}\left(\phi_{1}^{\prime \prime}-\phi_{1}^{\prime}\right) S_{\alpha} S_{\beta} C_{\gamma}+2 a K_{\mathrm{e}}\left[S_{\alpha}\left(C_{\alpha}+C_{\beta} C_{\gamma}\right)+\frac{1}{8} S_{2 \alpha}\left(C_{2 \beta}+C_{2 \gamma}\right)\right] \\
& \times\left[S_{\beta}\left(C_{\beta}+C_{\alpha} C_{\gamma}\right)+\frac{1}{8} S_{2 \beta}\left(C_{2 \alpha}+C_{2 \gamma}\right)\right] .
\end{aligned}
$$

In Eqs. (7) to (10) $\phi_{1}^{\prime \prime}, \phi_{1}^{\prime}, \phi_{2}^{\prime \prime}$ and $\phi_{2}^{\prime}$ are the force constants for the first and second neighbour interionic interactions. $C_{n \alpha}=\cos \left(2 n \pi a K_{\alpha}\right)$ and $S_{n \alpha}=$ $\sin \left(2 n \pi a K_{\alpha}\right), K_{\alpha}, K_{\beta}$ and $K_{\gamma}$ are the Cartesian components of the phonon wave vector, $2 a$ is the lattice parameter.

The phonon frequencies are computed by solving the secular determinant, $\left|D_{\alpha \beta}-m \omega^{2} I_{\alpha \beta}\right|=0$, where $m$ is the ionic mass, $I$ is unit matrix, $\omega$ is the angular frequency.

The crystal equilibrium condition of Upadhyaya et al. [8] is given by

$$
\frac{\mathrm{d} \phi}{\mathrm{d} \Omega}=0
$$

or

$$
\begin{aligned}
& \frac{\mathrm{d} \phi^{\mathrm{ii}}}{\mathrm{d} \Omega}+\frac{\mathrm{d} \phi^{\text {ie }}}{\mathrm{d} \Omega}=0, \\
& \frac{\mathrm{d} \phi^{\mathrm{ii}}}{\mathrm{d} \Omega}=\frac{\mathrm{d}}{\mathrm{d} \Omega}\left[4 \frac{\mathrm{d} \phi_{1}}{\mathrm{~d} \Omega}+3 \frac{\mathrm{d} \phi_{2}}{\mathrm{~d} \Omega}\right]=\frac{1}{a}\left[\phi_{1}^{\prime}+\phi_{2}^{\prime}\right],
\end{aligned}
$$




$$
\frac{\mathrm{d} \phi^{\mathrm{ie}}}{\mathrm{d} \Omega}=-p_{\mathrm{e}}=\frac{3}{5} K_{\mathrm{e}} .
$$

From Eqs. (11) to (14), we have

$$
K_{\mathrm{e}}=\frac{5}{3 a}\left[\phi_{1}^{\prime}+\phi_{2}^{\prime}\right] \text {. }
$$

By extending the total dynamical matrix in the long wavelength limit, we get

$$
\begin{aligned}
& C_{11}=\frac{1}{3 a}\left(\phi_{1}^{\prime \prime}+\phi_{1}^{\prime}+\phi_{2}^{\prime \prime}\right)+\frac{25}{8} K_{\mathrm{e}}, \\
& C_{12}=\frac{1}{3 a}\left(\phi_{1}^{\prime \prime}-4 \phi_{1}^{\prime}-3 \phi_{2}^{\prime}\right)+\frac{25}{8} K_{\mathrm{e}}, \\
& C_{44}=\frac{1}{3 a}\left(\phi_{1}^{\prime \prime}+2 \phi_{1}^{\prime}+3 \phi_{2}^{\prime}\right) .
\end{aligned}
$$

Substitution of Eq. (15) in (16) to (17) eliminates one independent model parameter. It is interesting to note that Sarkar et al. [6] and Das et al. [3] gave the expression for pressure of the electron gas in terms of the model parameters $\phi_{1}^{\prime}$ and $\phi_{2}^{\prime}$ and did utilize to eliminate one independent parameter by equating the pressure to the bulk modulus by $p_{\mathrm{e}}=\frac{3}{5} K_{\mathrm{e}}$ and putting in the expression of the total elastic constants. They rather introduced $p_{e}$ as a new parameter. The practice is not recommended by the researcher in lattice dynamics.

\section{Numerical computation}

We have four independent parameters in the present model and in order to evaluate them numerically we have utilized the expressions relating these parameters with three elastic constants and one longitudinal frequency of phonon wave at the zone boundary of the $[\xi, 0,0]$. The input data and the output result of the force constants are given in Table I and Table II. While the elastic constants of

\section{TABLE I}

Input values of the experimental data.

\begin{tabular}{l|c|c|c|c|c}
\hline \hline Metal & $\begin{array}{c}\nu_{\mathrm{L}}(\xi, 0,0) \\
{\left[10^{12} \mathrm{~Hz}\right]}\end{array}$ & $\begin{array}{c}\nu_{\mathrm{L}}(\xi, \xi, 0) \\
{\left[10^{12} \mathrm{~Hz}\right]}\end{array}$ & $\begin{array}{c}C_{11} \\
{\left[10^{11} \mathrm{~d} / \mathrm{cm}^{2}\right]}\end{array}$ & $\begin{array}{c}C_{12} \\
{\left[10^{11} \mathrm{~d} / \mathrm{cm}^{2}\right]}\end{array}$ & $\begin{array}{c}C_{44} \\
{\left[10^{11} \mathrm{~d} / \mathrm{cm}^{2}\right]}\end{array}$ \\
\hline Sodium & 3.58 & 3.82 & 0.808 & 0.664 & 0.586 \\
Potassium & 2.21 & 2.40 & 0.4167 & 0.3413 & 0.286 \\
Lithium & 8.82 & 9.00 & 1.484 & 1.253 & 1.080 \\
Rubidium & 1.325 & 1.46 & 3.14 & 2.64 & 1.89 \\
Caesium & 0.92 & 1.05 & 0.247 & 0.206 & 0.148
\end{tabular}


TABLE II

Output values of the atomic force constants.

\begin{tabular}{l|c|c|c|c}
\hline \hline \multicolumn{1}{c|}{ Metal } & $\phi_{1}^{\prime \prime}\left[10^{3} \mathrm{~d} / \mathrm{cm}\right]$ & $\phi_{1}^{\prime}\left[10^{3} \mathrm{~d} / \mathrm{cm}\right]$ & $\phi_{2}^{\prime \prime}\left[10^{3} \mathrm{~d} / \mathrm{cm}\right]$ & $\phi_{2}^{\prime}\left[10^{3} \mathrm{~d} / \mathrm{cm}\right]$ \\
\hline Sodium & 3.8020 & -0.0909 & 0.4871 & 0.0978 \\
Potassium & 2.5989 & -0.1261 & 0.4492 & 0.1171 \\
Lithium & 6.3177 & -0.0104 & 0.3826 & 0.0210 \\
Rubidium & 2.0154 & -0.0971 & 0.3713 & 0.0946 \\
Caesium & 1.6689 & -0.0015 & 0.1084 & 0.0105
\end{tabular}

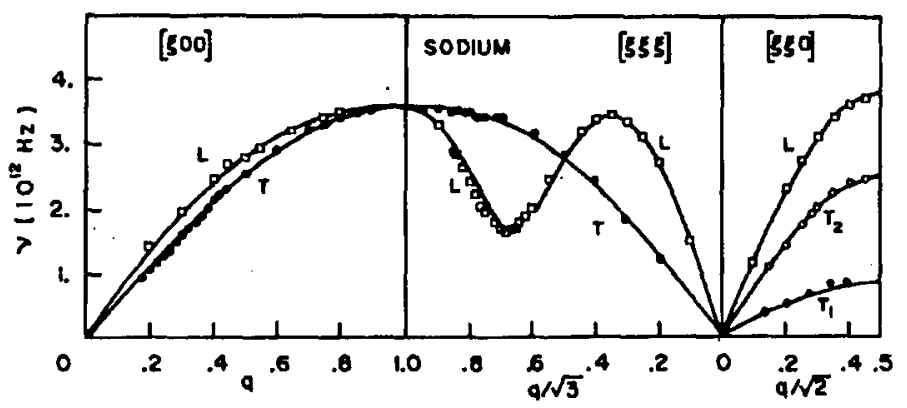

Fig. 1. Phonon dispersion relations in sodium along $[\xi 00]$, $[\xi \xi 0]$ and $[\xi \xi \xi]$ directions. Solid lines represent present calculations, $\square, \circ$, $\bullet$ represent experimental points.

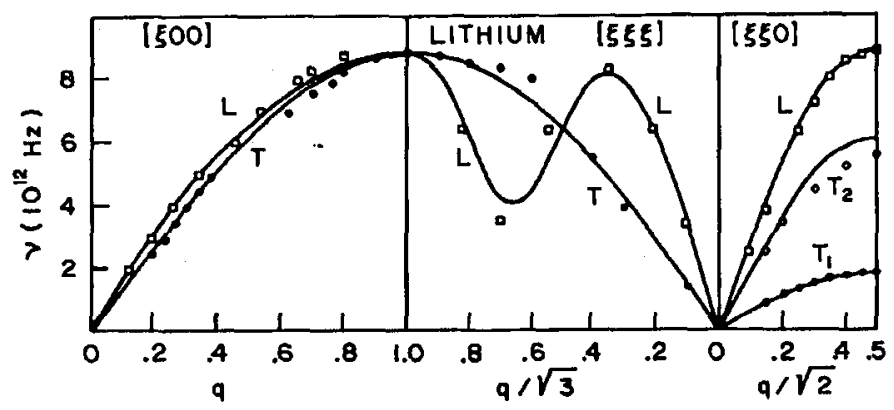

Fig. 2. Phonon dispersion relations in lithium along $[\xi 00],[\xi \xi 0]$ and $[\xi \xi \xi]$ directions. Captions are the same as for Fig. 1.

sodium, potassium, caesium, lithium and rubidium were taken respectively from the papers of Quimby and Siegal [9], Marquardt and Trivisonno [10], Kollarits and Trivisonno [11], Nash and Smith [12] and Gutman et al. [13], the experimental values of phonons respectively for sodium, potassium, caesium, lithium and rubidium 


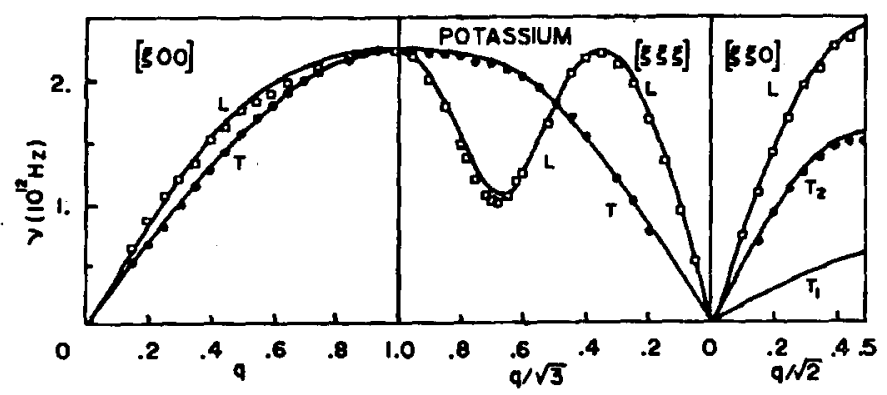

Fig. 3. Phonon dispersion relations in potassium along $[\xi 00],[\xi \xi 0]$ and $[\xi \xi \xi]$ directions. Captions are the same as for Fig. 1.

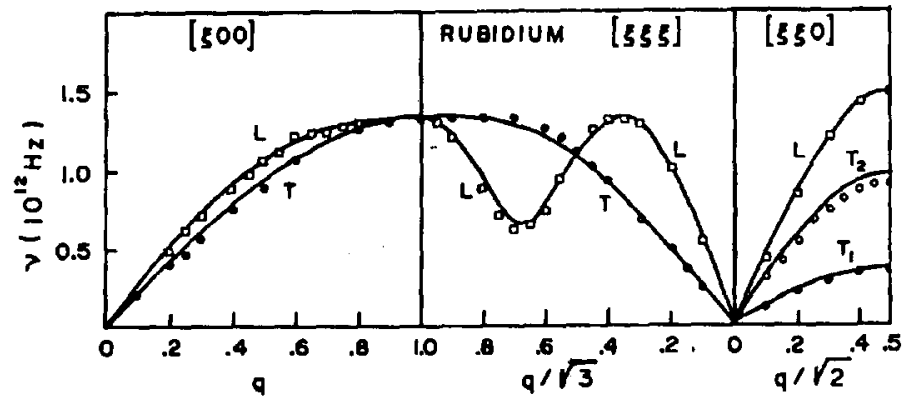

Fig. 4. Phonon dispersion relations in rubidium along $[\xi 00],[\xi \xi 0]$ and $[\xi \xi \xi]$ directions. Captions are the same as for Fig. 1.

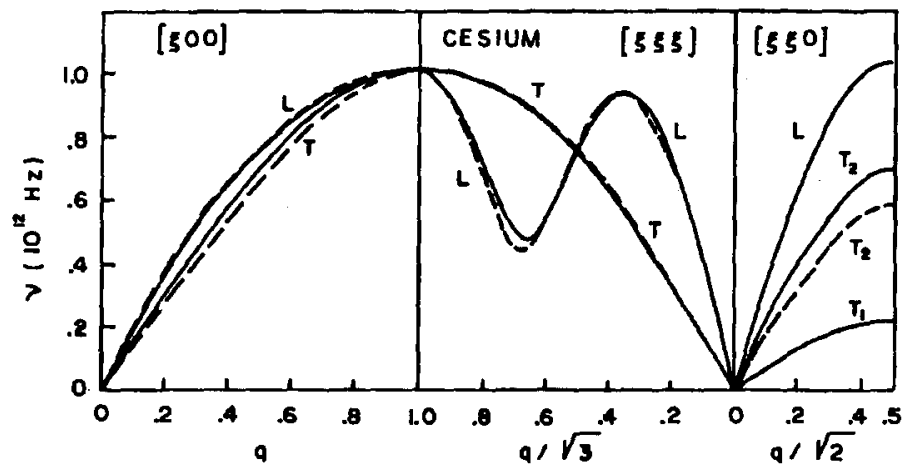

Fig. 5. Phonon dispersion relations in caesium along [ $\xi 00]$, $[\xi \xi 0]$ and $[\xi \xi \xi]$ directions. Solid lines represent present calculations and broken lines represent experimental curves.

were taken from the papers of Woods et al. [14], Cowley et al. [15], Nücker and Buchenau [16], Smith et al. [17] and Copley et al. [18].

The numerical values of force constants were used to compute phonon dispersion relations along the principal symmetry directions i.e. $[\xi 00],[\xi \xi 0]$ and $[\xi \xi \xi]$ and 

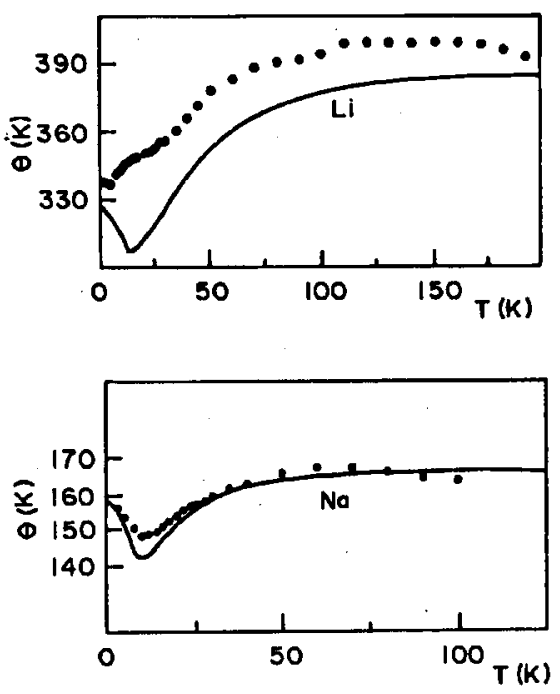

Fig. 6. $\theta-T$ curves of lithium and sodium. Solid lines represent present calculations and experimental points are shown by $\bullet$.
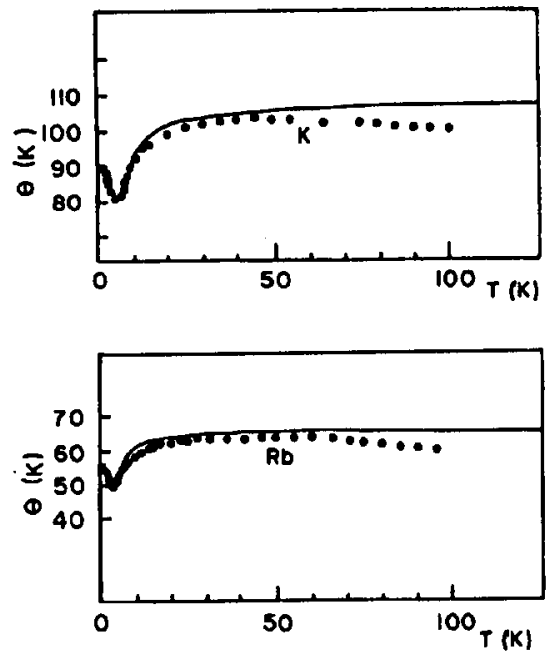

Fig. 7. $\theta-T$ curves of potassium and rubidium. Captions are the same as for Fig. 6.

also for irreducible wave vectors of phonons in the first Brillouin zone by solving the secular determinant (10) and (11). In Figs. 1 to 5 there are shown the computed and experimental phonons of $\mathrm{Na}, \mathrm{K}, \mathrm{Ce}, \mathrm{Li}$ and $\mathrm{Rb}$. The phonon density of states i.e. $\mathrm{g}(\nu)$ versus $\nu$ curves were plotted by utilizing the total phonon spectrum in the Brillouin zone, i.e. 24000 frequencies. The width of the frequency was taken 


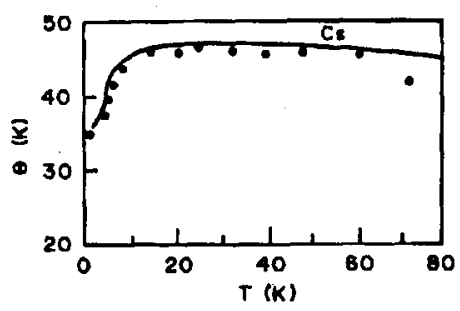

Fig. 8. $\theta-T$ curves of caesium. Captions are the same as for Fig. 6 .

to be $\Delta \nu=0.05 \times 10^{12} \mathrm{~s}^{-1}$. The specific heat at constant volume was calculated from a knowledge of the $g(\nu)$ versus $\nu$ curves from Blackman [19] technique. The corresponding $\theta-T$ curves for all the five metals are shown in Figs. 6 to 8 .

\section{Comparison with experimental results}

\subsection{Sodium}

A critical study of Fig. 1 reveals that there is close agreement between the computed and experimental phonons for sodium for almost all wave vectors in all the three principal symmetry directions. There is a bit discrepancy between the calculated and experimental phonons in the transverse branch $T_{1}$ of $[\xi \xi 0]$ and transverse branch of $[\xi \xi \xi]$ directions. But, in no way, the difference between the computed and experimental frequency is more than 5\%. A look at Fig. 6 shows that there is good agreement between calculated and experimental $\theta-T$ curves. The computed curve almost reproduced the experimental one at high temperatures. At low temperature the computed values of $\theta$ are lower by $4 \%$. The experimental $\theta$ was taken from the paper of Martin [20].

\subsection{Potassium}

A close study of Fig. 3 gives that there is excellent agreement between the computed and experimental phonons along all the three symmetry directions. For the transverse branch $T_{1}$ for the [ $\left.\xi \xi 0\right]$ direction we have no measurement of the experimental phonons. Our calculated phonons show a small deviation from the measured phonons for a high phonon wave vector in the longitudinal and transversal branches of $[\xi 00]$ and $[\xi \xi 0]$ but in no case the calculated phonon is off by more than $8 \%$.

A study of Fig. 7 shows that the calculated $\theta-T$ curve reproduces the entire course of the experimental work but lies above the experimental curve by $3 \%$. The experimental $\theta$ was taken from the paper of Filby and Martin [21].

\subsection{Rubidium}

A look at Fig. 4 shows that there is excellent agreement between the computed and experimental phonons in all the three principal symmetry directions. 
The theoretical phonon frequencies in the $T_{2}$ branch of $[\xi \xi 0]$ are about 3 to 8 percent higher than the experimental frequencies.

A study of Fig. 7 gives that there is excellent agreement between the computed and experimental $\theta-T$ curves. The computed curve is about $2 \%$ above the experimental one. The experimental $\theta$ was taken from the paper of Martin [20].

\subsection{Lithium,}

A look at Fig. 2 shows that there is excellent agreement between the computed and experimental phonons in lithium along all the three principal symmetry directions. Our calculated results along the $[\xi 00]$ direction have also shown the phonon anomaly i.e. crossing of longitudinal and transversal branches. We have not been able to reproduce well the experimental transverse branch of $[\xi \xi 0]$ direction. The maximum deviation between the calculated and experimental phonon is of the order of $7 \%$.

A study of Fig. 6 gives that the computed $\theta-T$ curve of lithium has reproduced entire course of the experimental curve. Our theoretical curve lies about $3 \%$ below the experimental one. The experimental $\theta$ was taken from the paper of Filby and Martin [21].

\subsection{Caesium}

A look at Fig. 5 shows that there is good agreement between the calculated and experimental phonons in caesium along all the three symmetry directions. Except for the transverse branch of $[\xi \xi 0]$ direction, where the theoretical curve is about $10 \%$ above the experimental one, our calculated phonons have almost reproduced the experimental one. It is surprising to note that other theoretical workers, such as Singh et al. [22] also have not been able to fit the $T_{2}$ branch of $[\xi \xi 0]$ direction.

A look at Fig. 8 demonstrates that our theoretical $\theta-T$ curve has reproduced the entire course of experimental one. The computed curve lies about $2 \%$ above the experimental curve. The experimental $\theta$ was taken from the paper of Filby and Martin [23].

\section{Discussion and conclusion}

We have investigated the lattice dynamics and thermal property of five alkali metals in a phenomenological model of Das et al. [3] after modifying it in extending electron-ion interaction and including the crystal equilibrium condition. Our results have shown with no doubt a definite improvement over the work of Das et al. [3] and Sarkar et al. [6] on alkali metals. It should be noted that these authors studied alkali metals two times. In first attempt they considered the electron-ion interaction effective between an atom and its first neighbours. In second attempt they extended electron-ion interaction out to second neighbours but unfortunately they committed an error in their work. We extended the electron-ion interaction out to third neighbours. The effect of introducing higher neighbour electron-ion 
interaction plays a vital role in determining the model parameters. This fact can be explained mathematically by showing that

$$
\begin{aligned}
& C_{11 \text { experimental }}=C_{11 \text { ionic }}+C_{11 \text { electronic }} \\
& C_{12 \text { experimental }}=C_{12 \text { ionic }}+C_{12 \text { electronic }} \\
& C_{44 \text { experimental }}=C_{44 \text { ionic }}+C_{44 \text { electronic }}
\end{aligned}
$$

If we confine the electron-ion interaction out to first neighbour

$$
C_{1 \text { experimental }}=C_{12 \text { ionic }}+K_{\mathrm{e}} \text {. }
$$

If the electron-ion interaction is out to second neighbours

$$
C_{11 \text { electronic }}=C_{12 \text { electronic }}+2 K_{\mathrm{e}} \text {. }
$$

If the electron-ion interaction is out to third neighbours

$$
C_{11 \text { electronic }}=C_{12 \text { electronic }}+\frac{25}{8} K_{\mathrm{e}} \text {. }
$$

We are sure that Sarkar et al. [6] wanted to keep the expression of $C_{11 \text { electronic }}=C_{12 \text { electronic }}$ to be the same for the first and second neighbours equal to $K_{\mathrm{e}}$ in accordance with the model of de Launay [2], Bhatia [24] and Krebs [25], that is why they divided by $1 / 2$ their Eq. (4). They forgot that in their model $K_{\mathrm{e}}$ depends on $\left(\chi^{l}\right)^{2}$. The different expressions for electronic interaction of the elastic constants guarantees a different value of $\phi_{1}^{\prime}, \phi_{1}^{\prime \prime}, \phi_{2}^{\prime \prime}$ and $\phi_{2}^{\prime \prime}$. Also the use of crystal equilibrium condition reduces one model parameter. We extended electron-ion interaction out to fourth and fifth neighbours also. These results did not show any improvement obtained over the third neighbour interaction.

We have shown here that the elastic constants of the alkali metals depend on the ionic potential plus a volume dependent potential in three body interaction. Our volume dependent potential is not of the nature of long range as is in the pseudopotential scheme of Brovman and Kagan [28]. Eventually for a better description of elastic constants of metals we should also consider the anharmonic ionic potential as has been carried out in the paper of Cowley [29].

The present work on alkali metals with only four adjustable parameters for the five alkali metals, sodium, potassium, rubidium, lithium and caesium gave a better description in comparison to the six parameter study of these metals by Sarkar et al. [6]. For caesium these authors have not made such a study.

In conclusion we can say that the present study of lattice dynamics and thermal property of alkali metals in a four parameter phenomenological model gave excellent agreement with the experimental findings. This phenomenological model has an essential feature of a model on first principles, i.e. preserving the crystal symmetry in the calculated phonons in reciprocal space. Also results predicted by this model are in no way inferior to other published results so far either on phenomenological models (Mishra and Singh, [26] and references therein) or on pseudopotential technique (Singh et al. [22] and Sen et al., [27] and references therein).

\section{Acknowledgments}

The authors are thankful to the referee for pointing out the references of Brovman, Kagan and Cowley. 


\section{References}

[1] T. Toya, J. Res. Inst. Catalysis, Hokkaido Univ. 7, 60 (1969).

[2] J. de Launay, Solid State Physics, Vol. 2, Ed. Seitz, A. Turnbull, Academic, New York 1956, p. 220.

[3] S.K. Das, D. Roy, S. Sengputa, Pramana 8, 117 (1977).

[4] S.K. Sarkar, S.K. Das, S. Sengputa, Phys. Status Solidi B 83, 615 (1977).

[5] B. Dutta, S.K. Sarkar, D. Roy, Phys. Status Solidi D 132, 345 (1985).

[6] S.K. Sarkar, S.K. Das, D. Roy, Acta Phys. Pol. A 55, 521 (1979).

[7] M. Lax, in: Proc. Int. Conf. on Lattice Dynamics, Copenhagen 1963, Ed. R.F. Wallis, Pergamon Press, 1965, p. 179.

[8] J.C. Upadhyaya, S.S. Sharma, O.P. Kulshrestha, Phys. Rev. B 12, 2236 (1975).

[9] S.L. Quimby, S. Siegal, Phys. Rev. 54, 293 (1938).

[10] W.R. Marquardt, J. Trivisonno, J. Phys. Chem. Solids 26, 273 (1965).

[11] F.J. Kollarits, J. Trivisonno, J. Phys. Chem. Solids 29, 2133 (1968).

[12] C.H. Nash, S.C. Smith, J. Phys. Chem. Solids 9, 113 (1959).

[13] E.J. Gutman, J. Trivisonno, J. Phys. Chem. Solids 28, 805 (1967).

[14] A.D.B. Woods, B.N. Brockhouse, R.H. March, A.T. Stewart, R. Bowers, Phys. Rev. 128, $1112(1962)$.

[15] R.A. Cowley, A.D.B. Woods, G. Dolling, Phys. Rev. 150, 487 (1966).

[16] N. Nücker, U. Buchenau, Phys. Rev. 31, 5479 (1985).

[17] H.G. Smith, G. Dolling, R.M. Nicklow, in: Proc. Int. Conf. on Inelastic Neutron Scattering, Vol. 1, I.A.E.A., Vienna 1962, p. 149.

[18] J.R.D. Copley, B.N. Brockhouse, S.H. Chem, in: Proc. Int. Conf. on Inelastic Neutron Scattering, Vol. 1, I.A.E.A., Vienna 1968, p. 209.

[19] M. Blackman, Proc. R. Soc. A 159, 416 (1937).

[20] D.L. Martin, Proc. R. Soc. A 254, 433 (1960).

[21] J.D. Filby, D.L. Martin, Proc. R. Soc. A 276, 187 (1963).

[22] N. Singh, N.S. Banger, S.P. Singh, Phys. Rev. 39, 3097 (1989).

[23] J.D. Filby, D.L. Martin, Proc. R. Soc. A 284, 83 (1965).

[24] A.B. Bhatia, Phys. Rev. 97, 363 (1955).

[25] K. Krebs, Phys. Rev. A 138, 143 (1965).

[26] S.K. Mishra, T.N. Singh, Phys. Status Solidi B 158, 153 (1990).

[27] D. Sen, S.K. Sarkar, S.K. Das, D. Roy, S. Sengputa, Acta Phys. Pol. A 57, 73 (1980).

[28] E.G. Brovman, Yu.M. Kagan, Sov. Phys. Usp. 112, 125 (1974).

[29] R.A. Cowley, Adv. Phys. 12, 421 (1963). 